\title{
Perfil epidemiológico das ceratomicoses atendidas no HC-UNICAMP
}

\author{
Epidemiological profile of keratomycosis at the HC-UNICAMP
}

\author{
Guilherme Gubert Müller ${ }^{1}$, Newton Kara-Josép ${ }^{1}$, Rosane Silvestre de Castro ${ }^{1}$
}

\section{RESUMO}

Introdução: A ceratite fúngica é uma importante causa de infecção corneana principalmente em países tropicais. Com alta morbidade devido à demora no diagnóstico, o exame laboratorial torna-se obrigatório nesses casos. Dificuldades técnicas na obtenção das amostras, porém, geram um grande número de exames falso-negativos. Nesses casos, uma detalhada avaliação do paciente em busca de características específicas dessa entidade pode auxiliar na elucidação diagnóstica. Objetivos: Formar um perfil epidemiológico dos portadores de ceratomicose para auxiliar no diagnóstico e tratamento precoces.

Métodos: Realizou-se estudo retrospectivo em base de dados de portadores de úlceras corneanas em busca de pacientes com diagnóstico de ceratomicose. Foram coletadas informações a respeito de sexo, situação do domicílio, grau de instrução, profissão, idade, fatores desencadeantes e predisponentes (oculares e sistêmicos), hipótese etiológica inicial, resultado laboratorial, tempo de cicatrização, complicações e tratamentos adjuvantes.

Resultados: Dos 599 pacientes atendidos por úlcera de córnea, 150 (25\%) foram diagnosticados como de etiologia fúngica. Houve maior prevalência em homens em idade produtiva e baixo nível de escolaridade. Trauma com material vegetal esteve presente em 74 (49,33\%) casos. Demais fatores identificados como trabalhadores e moradores de áreas rurais também suportam o trauma vegetal como principal fator de risco identificado no estudo.

Conclusões: Com base nos dados do estudo foi possível formar um perfil dos portadores de úlceras fúngicas atendidos pelo HC-UNICAMP.

Descritores: Infecções oculares fúngicas/epidemiologia; Micoses/epidemiologia; Ceratite; Úlcera da córnea

\section{ABSTRACT}

Background: Fungal keratitis is an important cause of corneal infection mainly in tropical countries. With high morbidity due to delay in diagnosis, laboratory examination is mandatory in these cases. However, technical difficulties in obtaining samples generate a large number offalse negatives. In these cases a detailed patient assessment for specific characteristics of this entity may help in diagnosis.

Purpose: To create an epidemiological profile of patients with keratomycosis to assist in early diagnosis and treatment.

Methods: A retrospectivestudy was performed by searching patients with keratomycosis diagnostic on the cornealulcers database. Information about gender, household location, educational level, occupation, age, predisposing and precipitating factors (ocular and systemic), initial etiological hypothesis, laboratory results, healing time, complications, and adjuvant treatments were collected.

Results: Of 599 patients with corneal ulcer, 150 (25\%) were diagnosed as fungal infection. There was a higher prevalence in males of working age and low educational level. Trauma with plant material was involved in 74 (49.33\%) cases. Other factors such as workers and residents of rural areas also supports trauma with vegetal as the main risk factor identified in the study.

Conclusions: Based on the collected data, it was possible to form a profile of patients with fungal ulcers treated by HC-UNICAMP.

Keywords: Eye infection, fungal/epidemiology; Mycosis/epidemiology; keratitis; Corneal ulcer

\section{INTRODUÇÃO}

Os fungos estão entre as principais causas de infecções corneanas em países de clima tropical. Devido à dificuldade no diagnóstico e tratamento, há altas taxas de morbidade ocular associadas. Estimase que entre 5 e $25 \%$ das ceratites infecciosas sejam causadas por fungos, sendo os fungos filamentosos os agentes mais comumente isolados em todo o mundo(1).

O aumento na incidência de casos deve-se, provavelmente, ao uso irrestrito de antibióticos de amplo espectro, que propiciam um ambiente de baixa competitividade, favorecendo o crescimento do fungo ${ }^{(2)}$. Outros fatores a serem considerados são o uso abusivo de corticoides tópicos, olho seco, lentes de contato, cirurgias oculares prévias, uso de medicações ou doenças imunossupressoras e doenças que alterem a flora ocular ${ }^{(3-7)}$.

Embora com características clínicas particulares, as infecções fúngicas geralmente confundem-se com as bacterianas, tornando a pesquisa etiológica laboratorial mandatória. Alguns autores recomendam a introdução de terapia antifúngica apenas após a confirmação laboratorial, com base na maior prevalência das infecções bacterianas ${ }^{(8)}$. Observamos na prática, porém, que há um alto número de exames falso-negativos, acarretando demora da introdução da terapia antifúngica adequada ${ }^{(9,10)}$.

Devido a essa dificuldade no diagnóstico laboratorial, a história clínica e o exame físico ganham especial importância. Características particulares das lesões fúngicas, tais como margens hifadas e irregulares, limites mal definidos, coloração acinzentada e lesões satélites associadas a dados de epidemiologia, como local de residência, predisposição a traumas, época do ano, idade, sexo e profissão, contribuem para a agilidade no diagnóstico ${ }^{(11,12)}$.

Esse estudo tem por objetivo formar um perfil epidemiológico dos portadores de ceratites fúngicas atendidos no Hospital de Clínicas da Universidade Estadual de Campinas (HC-UNICAMP) para auxiliar no diagnóstico e tratamento precoces.
Submetido para publicação: 21 de novembro de 2011

Aceito para publicação: 22 de julho de 2012

Trabalho realizado no Departamento de Oftalmologia, Faculdade de Ciências Médicas, Universidade Estadual de Capinas - UNICAMP - Campinas (SP), Brasil.

Médico, Departamento de Oftalmologia, Faculdade de Ciências Médicas, Universidade Estadual de Campinas - UNICAMP - Campinas (SP), Brasil.
Financiamento: Não houve financiamento para este trabalho.

Divulgação de potenciais Conflitos de Interesse: G.G.Müller, Nenhum; N.Kara-José, Nenhum; R.S.Castro, Nenhum.

Endereço para correspondência: Guilherme Gubert Müller. Av. Des. Hugo Simas, 333 - Curitiba PR - 80520-250 - Brasil - E-mail: guimuller@ig.com.br 


\section{MÉTODOS}

Realizou-se estudo retrospectivo no Ambulatório de Úlceras de Córnea da Oftalmológica do Hospital das Clínicas da UNICAMP incluindo todos os pacientes que apresentaram diagnóstico de ceratite fúngica no período de abril de 2003 a dezembro de 2010.

Após serem submetidos a anamnese e exame oftalmológico incluindo informações a respeito de: sexo, local de residência, grau de instrução, profissão, idade, fatores desencadeantes e predisponentes (oculares e sistêmicos); foi realizada coleta de material para identificação laboratorial do agente causal.

As amostras foram obtidas com paciente posicionado em lâmpada de fenda e olho anestesiado com 1 gota de propacaína 0,5\%. 0 debridamento da lesão foi feito com lâmina no 15 estéril e a semeadura do material em placas de ágar-sangue, ágar-chocolate, caldo BHI e ágar-Sabouraud, além de duas lâminas para Gram e Giemsa.

Até o resultado laboratorial, instituiu-se terapia antibiótica empírica imediatamente após coleta do material conforme apresentação clínica da lesão (hipótese etiológica inicial). Quando havia suspeita de etiologia fúngica, eram iniciados antifúngicos empíricos tópicos (natamicina $5 \%$ ou anfotericina B 0,15\%) associados ou não ao debridamento da lesão. O tratamento foi alterado conforme a resposta terapêutica ou resultado da pesquisa e cultura microbiológica.

Os pacientes recebiam alta do ambulatório quando havia cicatrização da lesão ou necessidade de tratamento cirúrgico complementar (transplante, recobrimento conjuntival ou evisceração). Ao fim do tratamento foram avaliados o tempo de cicatrização da lesão, a presença de complicações e a necessidade de tratamentos adjuvantes à terapia medicamentosa. Não foram incluídos neste estudo aqueles pacientes que abandonaram o tratamento.

A base de dados, a coleta e a análise das informações foram feitas com auxílio do programa Epilnfo ${ }^{\circledR}$ (Epilnfo 3.5.3 - Centers for Disease Control and Prevention, Atlanta, GA, USA).

Este estudo foi aprovado pelo Comitê de Ética em Pesquisa da Faculdade de Ciências Médicas da UNICAMP sob o protocolo no $780 / 2011$

\section{RESULTADOS}

No período estudado foram atendidos 599 olhos com úlcera corneana. Desses, 150 (25\%) pacientes apresentaram diagnóstico final de ceratite fúngica. Em relação ao sexo, $111(74 \%)$ pacientes eram do sexo masculino e 39 (26\%) do sexo feminino. Oitenta e oito pacientes $(58,66 \%)$ residiam em áreas urbanas e $62(41,33 \%)$ na zona rural. Em relação ao grau de instrução, 18 pacientes (12\%) eram analfabetos, $100(66,66 \%)$ tinham cursado o ensino fundamental completo, 28 $(18,66 \%)$ o ensino médio e $4(3,10 \%)$ possuíam curso superior. A profissão mais prevalente foi a de lavrador, correspondendo a 74 (49,33\%) pacientes (Tabela 1). A média da idade foi de 43 anos, variando entre 2 e 85 anos, com mediana de 43 anos.

\section{Tabela 1. Ocupação dos indivíduos}

\begin{tabular}{lcc}
\hline Profissão & Frequência & $\%$ \\
\hline Lavrador & 74 & 49,33 \\
Construtor & 12 & 8,00 \\
Informal e outros & 24 & 16,00 \\
Desempregado & 3 & 2,00 \\
Aposentado & 10 & 6,66 \\
Do lar & 16 & 10,66 \\
Estudante & 11 & 7,33 \\
\hline Total & 150 & 100,00 \\
\hline
\end{tabular}

Com relação à presença de fatores desencadeantes, o trauma ocular com material de origem vegetal esteve presente em 74 $(49,33 \%)$ pacientes. A maioria não apresentava fatores predisponentes associados, tanto oculares (132 pacientes, 88\%) como sistêmicos (143 pacientes, 95,33\%) (Tabela 2).

Dos 150 pacientes com diagnóstico de ceratite fúngica ao final do tratamento, 110 (73,33\%) tiveram essa mesma hipótese diagnóstica desde a primeira consulta, baseada em dados da anamnese e exame físico. Trinta e quatro $(22,66 \%)$ pacientes diagnosticados inicialmente como portadores de úlcera bacteriana tiveram diagnóstico alterado para etiologia fúngica durante o tratamento. O diagnóstico final de ceratite fúngica foi baseado no resultado laboratorial e/ou resposta terapêutica positiva aos antifúngicos.

O exame do raspado corneano foi positivo para fungo em 24 pacientes (17,6\%), sendo 20 culturas positivas para Fusarium spp. e 4 positivas para Aspergillus spp. Não houve casos com suspeita clínica ou confirmação laboratorial de ceratites por leveduras no período avaliado (Tabela 3).

Houve cicatrização da úlcera em 112 pacientes (74,6\%); 11 pacientes (7,33\%) evoluíram com perfuração, 10 (6,66\%) com descemetocele, 9 (6\%) com necrose corneana, 5 (4\%) com sinéquias anteriores ou posteriores e 3 (2,32\%) com comprometimento de câmara anterior e cristalino. Não houve casos de endoftalmite.

Entre os pacientes com cura da infecção o tempo médio de cicatrização das lesões foi de 28 dias, variando de 1 a 82 dias, com mediana de 27 dias.

Dos 112 pacientes em que houve cicatrização da lesão, 24 (16\%) utilizaram apenas antifúngico tópico e 88 (58,66\%) fizeram uso de

Tabela 2. Fatores de risco avaliados

\begin{tabular}{|c|c|c|}
\hline & Frequência & $\%$ \\
\hline \multicolumn{3}{|l|}{ Fatores desencadeantes } \\
\hline Nenhum & 62 & 41,33 \\
\hline Trauma vegetal & 74 & 49,33 \\
\hline Trauma inespecifico & 11 & 7,33 \\
\hline Lentes de contato & 1 & 0,66 \\
\hline Trauma químico & 2 & 1,33 \\
\hline Total & 150 & 100,00 \\
\hline \multicolumn{3}{|l|}{ Fatores predisponentes oculares } \\
\hline Nenhum & 132 & 88,00 \\
\hline Olho seco & 1 & 0,66 \\
\hline Glaucoma & 5 & 3,33 \\
\hline Cirurgia prévia & 5 & 3,33 \\
\hline Uso crônico de colírios & 1 & 0,66 \\
\hline Edema crônico & 0 & 0,00 \\
\hline Lagoftalmo & 1 & 0,66 \\
\hline Herpes ocular prévio & 3 & 2,00 \\
\hline Transplante de córnea prévio & 2 & 1,33 \\
\hline Total & 150 & 100,00 \\
\hline \multicolumn{3}{|l|}{ Fatores predisponentes sistêmicos } \\
\hline Nenhum & 143 & 95,33 \\
\hline Diabetes & 6 & 4,00 \\
\hline Imunossupressão & 1 & 0,66 \\
\hline Doenças neurológicas & 0 & 0,00 \\
\hline Doenças reumáticas & 0 & 0,00 \\
\hline Total & 150 & 100,00 \\
\hline
\end{tabular}


colírio associado ao debridamento da lesão, a fim de favorecer a penetração da droga. Trinta e oito (26\%) necessitaram intervenções cirúrgicas, sendo o transplante de córnea o mais indicado (27 pacientes, 18\%) (Tabela 4).

\section{DISCUSSÃO}

Ceratites fúngicas ainda são um desafio na prática oftalmológica e, devido à baixa prevalência da doença, poucos são os estudos envolvendo grandes amostras. Hospitais de referência, como o HCUNICAMP, conseguem agrupar um grande número desses casos pela sua abrangência regional. Além disso, o hospital é referência de uma grande área da economia agrícola, com lavouras pouco mecanizadas, que utilizam maior mão de obra humana, aumentando o risco de traumas, entre eles o trauma ocular com material de origem vegetal(2).

A maior prevalência se deu em pacientes do sexo masculino com baixo grau de instrução e trabalhadores da zona rural, categorias tradicionalmente associadas à maior exposição a traumas. Além disso, o maior número de casos em indivíduos em idade produtiva acarreta maior impacto econômico às famílias desses indivíduos ${ }^{(13,14)}$.

No Brasil 15\% da população reside em zonas rurais. Entretanto, em nosso estudo, a parcela dessa população foi consideravelmente maior (62 pacientes - 41,33\%), mesmo que ainda inferior à população urbana (88 pacientes $-58,66 \%)^{(15)}$.

O grande número de traumas oculares com material vegetal associado confirma esse como o principal fator de risco identificado no estudo. Com nexo causal já bem estabelecido, o trauma vegetal

Tabela 3. Resultado do exame laboratorial

\begin{tabular}{lcc}
\hline Patógeno & Frequência & $\%$ \\
\hline Aspergillus spp & 4 & 3,10 \\
Fusarium spp & 20 & 15,50 \\
Bacillus spp & 3 & 2,32 \\
Micrococcus spp & 1 & 0,77 \\
Streptococcus pneumoniae & 1 & 0,77 \\
Staphycoccus aureus & 4 & 3,10 \\
Staphylococcus epidermidis & 11 & 8,52 \\
Streptococccus viridians & 2 & 1,55 \\
Staphylococcus spp & 3 & 2,32 \\
Não identificado & 80 & 62,01 \\
\hline Total & 129 & 100,00 \\
\hline
\end{tabular}

Tabela 4. Tratamento adjuvante

\begin{tabular}{lcc}
\hline Opção terapêutica & Frequência & $\%$ \\
\hline Nenhum & 24 & 16,00 \\
Debridamento & 88 & 58,66 \\
Recobrimento conjuntival & 7 & 4,66 \\
Evisceração & 0 & 0,00 \\
Cola de cianoacrilato & 2 & 1,33 \\
Membrana amniótica & 0 & 0,00 \\
Transplante de córnea & 27 & 18,00 \\
Lavagem de câmara anterior & 2 & 1,33 \\
\hline Total & 150 & 100,00 \\
\hline
\end{tabular}

é citado em diversos estudos como um importante dado na história clínica de lesões corneanas suspeitas de infecções fúngicas ${ }^{(2,16,17)}$.

A baixa incidência de infecções por leveduras pode ser a razão do baixo índice de fatores predisponentes encontrados no estudo ${ }^{(4,7)}$. Por outro lado, a alta incidência de fungos filamentares encontrados coincide com estudos que mostram este sendo mais comuns em pacientes hígidos e sem doença ocular prévia ${ }^{(18-20)}$.

Obtivemos baixa positividade dos exames laboratoriais, em comparação a estudos semelhantes ${ }^{(16,21,22)}$. Sabemos que a obtenção de agentes viáveis para cultura nas úlceras corneanas é sempre um desafio devido à escassez de material. No caso de úlceras fúngicas o desafio é ainda maior, pois esses penetram mais facilmente nas camadas profundas do estroma corneano, por diversas vezes, sem perda da estrutura do estroma anterior. Tal característica pode ser confirmada pela maior positividade de biópsias de córnea que de raspados em diversos estudos ${ }^{(23-25)}$. Em nosso serviço a coleta do material é feita por médicos residentes, acreditamos que a falha na técnica também contribua para o maior número de exames falso negativos. O grande número de casos com melhora clinica usando antifúngico que apresentaram cultura positiva para bactérias também pode ser atribuído à contaminação do material da coleta com micro-organismos da flora palpebral.

Cento e doze pacientes foram diagnosticados e tratados com sucesso apenas com dados clínicos, reforçando a importância de uma história e exame clínico minuciosos. Ao fazer o diagnóstico e iniciar o tratamento adequado precocemente diminuímos a perda tecidual e a penetração do agente e, consequentemente, o tempo de tratamento e a chance de sequelas são reduzidos.

O tempo médio de cicatrização no estudo também foi semelhante aos da literatura ${ }^{(11,12)}$.

Cerca de $26 \%$ evoluíram com complicações, número baixo quando comparado a outros estudos, com a maioria dos casos obtendo resolução da infecção com o tratamento clínico baseado nos antifúngicos tópicos e debridamento da lesão(26). Acreditamos que isso se deve a introdução precoce da terapia antifúngica baseada nos dados epidemiológicos da historia e características clínicas do paciente ao invés do aguardo pelo resultado laboratorial.

Os casos com evolução desfavorável geralmente estavam relacionados com o maior tempo de evolução da doença e a demora no diagnóstico e início da terapia antifúngica. Prova disso, cerca de $1 / 3$ dos transplantes foram indicado no momento da chegada do paciente ao serviço devido ao avançado quadro infeccioso.

Ao fim conseguimos formar um perfil dos portadores de úlceras fúngicas atendidos no HC-UNICAMP. Confirmamos a forte associação com o trauma vegetal e as condições que aumentam o seu risco: lavradores, moradores de zona rural e sexo masculino em idade produtiva; todos importantes fatores para o desenvolvimento da doença. Além disso, destaca-se a importância do exame clínico e a introdução precoce de terapia antifúngica frente às dificuldades laboratoriais.

\section{REFERÊNCIAS}

1. Wilhelmus K. Epidemiology of ocular infections In: Tasman W JE, editor. Foundations of Clinical Ophthalmology. Philadelphia: Lippincott-Raven; 1998.

2. Bharathi MJ, Ramakrishnan R, Meenakshi R, Shivakumar C, Raj DL. Analysis of the risk factors predisposing to fungal, bacterial \& acanthamoeba keratitis in south India. Indian J Med Res. 2009;130(6):749-57.

3. Rippon J. Medical Mycology. The Pathogenic Fungi and the Pathogenic Actinomycetes. Philadelphia: Saunders Co; 1988.

4. Höfling-Lima AL, Forseto A, Duprat JP, Andrade A, Souza LBd, Godoy P, et al. Estudo laboratorial das micoses oculares e fatores associados às ceratites. Arq Bras Oftalmol. 2005;68(1):21-7.

5. Kurokawa CS, Sugizaki MF, Peracoli MT. Virulence factors in fungi of systemic mycoses. Rev Inst Med Trop Sao Paulo. 1998;40(3):125-35

6. Wong TY, Ng TP, Fong KS, Tan DT. Risk factors and clinical outcomes between fungal and bacterial keratitis: a comparative study. CLAO J. 1997;23(4):275-81.

7. Oliveira PR, Resende SM, Oliveira FC, Oliveira AC. Ceratite fúngica. Arq Bras Oftalmol. 2001;64(1):75-9. 
8. Allan BD, Dart JK. Strategies for the management of microbial keratitis. Br J Ophthalmol. 1995;79(8):777-86.

9. Kumar M, Shukla PK. Use of PCR targeting of internal transcribed spacer regions and single-stranded conformation polymorphism analysis of sequence variation in different regions of rrna genes in fungi for rapid diagnosis of mycotic keratitis. J Clin Microbiol. 2005;43(2):662-8.

10. Dunlop AA, Wright ED, Howlader SA, Nazrul I, Husain R, McClellan K, et al. Suppurative corneal ulceration in Bangladesh. A study of 142 cases examining the microbiological diagnosis, clinical and epidemiological features of bacterial and fungal keratitis. Aust N Z J Ophthalmol. 1994;22(2):105-10.

11. Jones D. Diagnosis and management of fungal keratitis. Duane's clinical ophthalmology. $5^{\text {th }}$ ed. Philadelphia: Lippincott Williams \& Wilkins; 2005.

12. Alfonso EC GA, Miller D. Fungal keratitis. In: Krachmer JH MM, Holland EJ, editor. Cornea: fundamentals, diagnosis and management. $3^{\text {th }}$ ed. New York: Mosby Elsevier; 2010.

13. Ibrahim MM, Vanini R, Ibrahim FM, Martins W de P, Carvalho RT, Castro RS, Rocha EM. Epidemiology and medical prediction of microbial keratitis in southeast Brazil. Arq Bras Oftalmol. 2011;74(1):7-12

14. Vanzzini Zago V, Manzano-Gayosso P. Hernández-Hernández F, Méndez-Tovar LJ, Gómez-Leal A, López Martínez R. [Mycotic keratitis in an eye care hospital in Mexico City]. Rev Iberoam Micol. 2010;27(2):57-61. Spanish.

15. IBGE. Distribuição percentual da população nos Censos Demográficos, segundo as Grandes Regiões, as Unidades da Federação e a situação do domicílio - 1960/2010.2010; Available from: http://www.ibge.gov.br/home/estatistica/populacao/censo2010/ tabelas_pdf/Brasil_tab_1_9.pdf.

16. Gopinathan U, Garg P, Fernandes M, Sharma S, Athmanathan S, Rao GN. The epide- miological features and laboratory results of fungal keratitis: a 10-year review at a referral eye care center in South India. Cornea. 2002;21(6):555-9.

17. Costa ML GP, Lage J. Flora micótica da conjuntiva de indivíduos normais. Rev Bras Oftalmol. 1975;34(2):199-206.

18. Bharathi MJ, Ramakrishnan R, Vasu S, Meenakshi R, Palaniappan R. Epidemiologica characteristics and laboratory diagnosis of fungal keratitis. A three-year study. Indian J Ophthalmol. 2003:51(4):315-21.

19. Xie L, Zhong W, Shi W, Sun S.. Spectrum of fungal keratitis in north China. Ophthalmology. 2006;113(11):1943-8.

20. Andrade AJ, Vieira LA, Höfling-Lima AL, Yu MC, Gompertz OF, Freitas D, et al. Laboratorial analyses of fungal keratitis in a University Service. Arq Bras Oftalmol. 2000; 63(1):59-63.

21. Green M, Apel A, Stapleton F. Risk factors and causative organisms in microbial keratitis. Cornea. 2008;27(1):22-7.

22. Saha S, Banerjee D, Khetan A, Sengupta J. Epidemiological profile of fungal keratitis in urban population of West Bengal, India. Oman J Ophthalmol. 2009;2(3):114-8.

23. Alexandrakis G, Haimovici R, Miller D, Alfonso EC. Corneal biopsy in the management of progressive microbial keratitis. Am J Ophthalmol. 2000;129(5):571-6.

24. Ishibashi Y, Hommura S, Matsumoto Y. Direct examination vs culture of biopsy specimens for the diagnosis of keratomycosis. Am J Ophthalmol. 1987;103(5):636-40.

25. Rosa RH, Jr., Miller D, Alfonso EC. The changing spectrum of fungal keratitis in south Florida. Ophthalmology. 1994;101(6):1005-13.

26. Salera CM, Tanure MA, Lima WT, Campos CM, Trindade FC, Moreira JA. Perfil das ceratites fúngicas no Hospital São Geraldo Belo Horizonte - MG. Arq Bras Oftalmol. 2002;65(1):9-13.

\section{${ }_{4}$ jornada Paulista de Oftalmologia \\ 4a Jornada Paulista de Oftalmologia}

22 e 23 de março de 2013

\section{Hotel Fonte Colina Verde}

São Pedro (SP)

Promoção:

UNESP

UNICAMP

USP - Ribeirão Preto

\section{Informações:}

JDE Eventos

Tels.: (11) 5084-9174 / 5082-3030 / 5084-5284

E-mail: diva@jdeeventos.com.br

Site: www.jdeeventos.com.br 